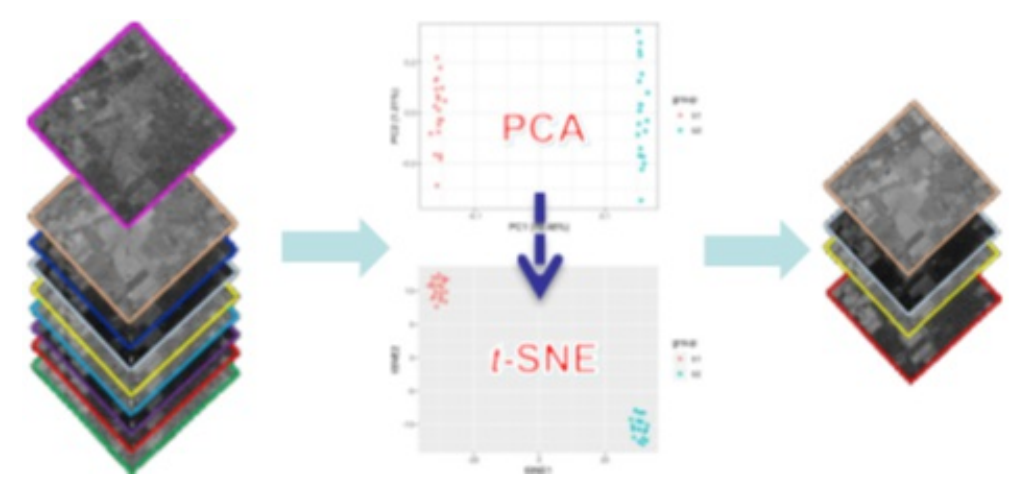

\title{
Spectral Graph Neural Networks with Manifold- Learning-Based Feature Extraction for Hyperspectral Image Classification
}

\author{
Shengliang $\mathrm{Pu}^{1}$ \\ 1 East China University of Technology (ECUT) \\ Funding: The author(s) received no specific funding for this work. \\ Potential competing interests: The author(s) declared that no potential competing interests exist.

\begin{abstract}
In this short article, we briefly retrospect the recent progress of spectral graph neural networks with manifold-learning-based feature extraction for hyperspectral image classification.
\end{abstract}

\section{Introduction}

Graph-based deep learning has shown superiority for resolving graph-structured data. In particular, graph representation learning (GRL) and graph neural networks (GNNs) have proved a promising approach with a plain superiority for learning graph data and modeling feature topological relations. Compared to deep learning represented by convolutional neural networks (CNNs), graph-based deep learning has the advantage of illustrating class boundaries and modeling feature relationships. In terms of a hyperspectral image classification task, hyperspectral images (HSIs) from regular grids into irregular domains can adapt to the advantages of graph-based deep learning. Therefore, the priority problem would be how to convert hyperspectral data into irregular domains from regular grid-like structures. 
The previous study (published) presented a novel method that performs localized graph filtering ${ }^{[1]}$ on HSIs based on spectral graph theory. ${ }^{[2]}$ First, we conducted the principal component analysis (PCA) preprocessing to create localized hyperspectral data cubes with unsupervised feature reduction (involving 10 principal components), and then combined with localized adjacent matrices, were fed into a lightweight graph convolution network in a standard supervised learning paradigm. Finally, we succeeded in analyzing diversified land covers by considering local graph structure with spectral graph filtering and using spatialspectral information. Accordingly, we draw the following beneficial conclusions.

- Experiments indicated that effective feature learning is beneficial to improving training efficiency of hyperspectral image classifiers and to enhance the final classification accuracy of deep learning or graph deep learning models.

- Adjacency matrix constructed by localized spectral filtering in reference with hyperspectral cubes will be refined in the training process of graph deep learning models, which makes GCN good applicability to the patch-based hyperspectral data.

- Standard supervised learning might be degraded compared to the semi-supervised learning paradigm which could take full advantage of a small number of labeled samples and a large number of unlabeled samples, yet it has a certain reference value for mining global graph structure information in case of combining with a full-graph adjacency matrix.

- Compared with classifiers such as CNN and support vector machine (SVM), GCN could achieve good classification performance, especially for complex hyperspectral data sets. Besides, it might have better convergence characteristics fitting a graph-based deep learning model.

- The final classification results of hyperspectral image classification not only depend on the employed classifiers or models, sampling process, and parameter selection, but also depend on the complexity and quality of the used data set.

- Preparation of hyperspectral data for feeding a graph-based deep learning model that draws from the previous strategy how to prepare HSI data for image-based deep learning, is a novel approach in terms of the existing publications.

\section{Graph attention network with t-SNE manifold learning}

The subsequent study (unpublished) proposed a novel approach that combines t-SNE (t-distributed stochastic neighbor embedding) ${ }^{[3]}$ manifold learning and graph attention network ${ }^{[4]}$ for the hyperspectral image classification task. First, t-SNE manifold learning was used to create localized hyperspectral patches rather than PCA statistical learning. Then, through a supervised learning way to train the designed graph attention network using these patch-based feature cubes and the equal-sized localized adjacent matrices. Finally, credible classification results and higher land cover mapping in distinguishing diversified land 
covers through reducing the redundancy of spectral information and enhancing the expression of local spatial-spectral information were obtained. There are several useful revelations that can be concluded here.

- GAT has achieved better classification accuracies than GCN in a computer vision task. Yet it might be slightly inferior to GCN in a hyperspectral image classification task, and requires about twice the training and evaluating time.

- Existing publications suggest that additional data denoising could be helpful for downstream hyperspectral image processing and analysis, but for hyperspectral image augmentation and classification tasks, adding additional noise to the raw or scaled data might alleviate the problem of over-smoothing a graph-based learning model.

- Whether GCN or GAT models, if both simply increase the number of processing layers, then faced with the problem of decreasing classification accuracy. In this regard, increasing the complexity of samples or adding residual functionality might be a solution, which indicates that patch-based hyperspectral data might be less complex in a sense.

- Newly hyperspectral data set "Huanghekou" [5] has poor separability between categories (21 classes). Yet better classification performance could be obtained. It indicates that class separability might not be a critical issue, and the spatial texture and contextual information might somehow reduce the sensitivity of spectral differences to the properties of land surface materials.

- For t-SNE-based feature learning contains a large number of iterative calculations that lead to huge computational cost, so we adopt GPU acceleration to derive feature data and save them to the storage medium, then load and resample them again, further to effectively avoid huge memory consumption resulting in no response from the remote cloud server.

- Experiments demonstrate that increasing the noise level of adjacency matrix could indeed improve its complexity, but for a graph-based node classification task using hyperspectral cubes, the final classification performance would possibly be adversely influenced to some extent.

\section{Conclusions}

Experiments on real hyperspectral data sets demonstrated that the presented graph-based deep learning methods offer promising classification performance compared with other popular competitors (i.e., machine learning classifiers and deep learning models), and also proves the great importance of combining spatial- and spectral features and enhancing feature separability for hyperspectral image classification. 


\section{References}

1. ^Michaël Defferrard, Xavier Bresson, Pierre Vandergheynst. (2016). Convolutional Neural Networks on Graphs with Fast Localized Spectral Filtering. NIPS 2016. arXiv.

2. `Shengliang Pu, Yuanfeng Wu, Xu Sun, Xiaotong Sun. (2021). Hyperspectral Image Classification with Localized Graph Convolutional Filtering. Remote Sensing, vol. 13 (3), 526. doi:10.3390/rs13030526.

3. ^Laurens van der Maaten, Geoffrey Hinton. (2008). Visualizing Data using t-SNE. Journal of Machine Learning Research, vol. 9 . Microtome Publishing.

4. `Petar Veličković, Guillem Cucurull, Arantxa Casanova, Adriana Romero, Pietro Liò, Yoshua Bengio. (2017). Graph Attention Networks. ICLR 2018. arXiv.

5. ^Leilei Jiao, Weiwei Sun, Gang Yang, Guangbo Ren, et al. (2019). A Hierarchical Classification Framework of Satellite Multispectral/Hyperspectral Images for Mapping Coastal Wetlands. Remote Sensing, vol. 11 (19), 2238. doi:10.3390/rs11192238. 